\title{
A Mixed E-B Finite Element Formulation for the analysis of Periodic Structures
}

\author{
Vemula Bhanu Naidu ${ }^{1}$, Dipanjan Gope ${ }^{2}$, and Sudhir Kamath ${ }^{1}$ \\ ${ }^{1}$ Microwave Tube Research and Development Centre, Bangalore 560013, India \\ ${ }^{2}$ Department of Electrical Communication Engineering, Indian Institute of Science, Bangalore, India 560012 \\ Email: vbhanunaidu@gmail.com, and dipanjan.gope@gmail.com
}

\begin{abstract}
The analysis of periodic structures with the Finite Element Method (FEM) has received a lot of attention in recent years. FEM formulates Maxwell's curl equations as either a second-order wave equation or two dual first-order (mixed) equations. Up until now the analysis of the periodic structures was primarily restricted to using the second-order wave equation. In this work, a new mixed vector finite element method for solving the coupled Maxwell's equations for the periodic boundary condition is presented. The mixed-order formulation gives a higher accuracy compared to the second-order for the same number of mesh elements. Numerical experiments are performed to validate the new formulation with analytical and commercial methods.
\end{abstract}

Keywords-Finite element method, Mixed vector Finite element method, Floquets theorem, Periodic boundary conditions.

\section{INTRODUCTION}

Traditionally, many numerical methods have been developed for electromagnetic analysis of periodic structures, starting from closed domain frequency selective surfaces to open domain microstrip phased arrays. Numerous papers have been published on this method, most of which deal with metallic periodic structures [1]-[2]. The use of unstructured grids in FEM makes it advantageous for solving the complex electromagnetic problems [3]-[6]. FEM primarily deal with Maxwell's curl equations as either a second-order wave equation i.e. Electric field (EE method) and Magnetic field (BB method) equations, or two dual first-order equations. Till now the analysis of the periodic structures was carried out by using the second-order wave equation. The first finite element method for periodic problems was implemented for electromagnetic scattering by a two-dimensional grating and for 3D was associated with electromagnetic scattering and radiation of a cavity-backed antenna array.

In this work, a new FEM formulation is proposed, with dual first-order equations for the analysis of periodic structure. The proposed mixed electric magnetic (E-B) FEM for the analysis of the periodic structures, relies on a discretisation approach based on differential forms [7] - [11]. The boundary value problem is first cast in the dual first-order equation form i.e. in E-B form, followed by enforcing the periodic boundary conditions on sides of the Floquet cell. The mixed order formulation gives a higher accuracy as compared to second-order methods for the same number of mesh elements.

The paper is organized as follows: in Section II, the proposed mixed-order FEM formulation is detailed. Numerical results are presented in section III. Section IV concludes the paper.

\section{3D FULL-WAVE FEM FORMULATION USING MIXED VECTOR BASIS}

An infinite antenna array can be analyzed using a single unit cell, where the field at one periodic surface of a unit cell is related to the field at the opposite parallel surface of the unit cell through a simple phase shift. The objective in this section is to derive the E-B FEM formulation that is appropriate for unit cell to numerically analyze infinitely periodic structures in the frequency domain.

\section{A. Finite Element Formulation}

The equations of electromagnetism considered here are written as field quantities in Maxwell's equations with a single frequency are harmonically oscillating functions, i.e., in the form of complex phasor notation. The $\varepsilon, \mu$ and $\sigma$ are assumed depending on material properties, which take into account anisotropic media and these matrices are considered to be symmetric, positive definite.

On the contrary, if only isotropic cases are considered, then $\varepsilon=\varepsilon I_{3}$ and $\mu=\mu I_{3}$, where $\varepsilon$ and $\mu$ are strictly positive scalar functions and $\mathrm{I}_{3}$ is the identity matrix of $\mathbb{R}^{3}$. Let the electric and magnetic fields and the electric and magnetic inductions are denoted by E, H, D and B respectively. Eliminating $\mathrm{H}$ and D from the Maxwell's system the equations for an open subdomain $\Omega$ of $\mathbb{R}^{3}$ can be written as

$$
\begin{aligned}
\varepsilon \mathrm{j} \omega \vec{E}-\nabla \times\left(\mu^{-1} \vec{B}\right) & =-\sigma \vec{E} \in \Omega \\
\mathrm{j} \omega \vec{B}+\nabla \times \vec{E} & =0 \in \Omega
\end{aligned}
$$

Without loss of generality, it can be assumed that the boundary enclosing the volume $\Omega$ are PEC boundary ( $\partial \Omega_{\mathrm{PEC}}$ ) and Periodic boundary ( $\left.\partial \Omega_{\mathrm{PER}}\right)$. The PEC boundary $\left(\partial \Omega_{\mathrm{PEC}}\right)$ can be expressed in initial conditions as:

$$
\begin{gathered}
\vec{E} \times v=0 \text { on } \partial \Omega_{P E C} \\
\vec{B} \cdot v=0 \text { on } \partial \Omega_{P E C}
\end{gathered}
$$

Here $v$ is the vector normal to the boundary $\partial \Omega_{\mathrm{PEC}}$. The variation formulation of 1 with 2 is to find $\mathrm{E}$ and $\mathrm{B}$, such that

$$
E(.)=\Phi \in H_{0}(\operatorname{curl}, \omega) ; B(.)=\Psi \in H_{0}(\operatorname{div}, \omega)
$$

Here $\Phi$ and $\Psi$ are the vector spaces for $\mathrm{E}$ and $\mathrm{B}$ field representation respectively. i.e., the electric field intensity $\mathrm{E}$ in terms of Whitney edge elements $, \mathrm{W}_{1}=1,2, \ldots, \mathrm{N}_{\mathrm{e}}$, and the magnetic field flux density $\mathrm{B}$ in terms of Whitney face elements $, \mathrm{W}_{2}=1,2, \ldots, \mathrm{N}_{\mathrm{f}}$. For any other choice of $\Psi$ the condition $\nabla \cdot \mathrm{B}=$ 0 is not satisfied. So, the appropriate definition is $\Psi=\mathrm{H}$ (div, $\Omega$ ), for which $\nabla \cdot \mathrm{B}=0$ [12]. By integrating the weighted (3) over the domain of interest and applying Galarkin method for 
domain decomposition yields the mixed order E-B scheme. The system of integral equations to be solved over the $3 \mathrm{D}$ domain are:

$$
\begin{gathered}
j \omega \iiint_{\Omega} \varepsilon \vec{\Phi} \cdot \vec{\Phi} d V-\iiint_{\Omega} \mu^{-1}(\nabla \times \vec{\Psi}) \cdot \vec{\Phi} d V=0 \\
j \omega \iiint_{\Omega} \vec{\Psi} \cdot \vec{\Psi} d V+\iiint_{\Omega}(\nabla \times \vec{\Phi}) \cdot \vec{\Psi} d V=0 \\
\forall \vec{\Phi} \in H_{0}(\operatorname{curl}, \Omega)=W_{i}^{1}, \forall \vec{\Psi} \in H_{0}(\operatorname{curl}, \Omega)=W_{i}^{2}
\end{gathered}
$$

\section{B. Imposing the boundary condition}

As the system has no boundary integrals involved, a boundary term is brought out by applying the vector identity of

$$
(\nabla \cdot(\mathrm{A} \times \mathrm{B})=\mathrm{B} \cdot(\nabla \times \mathrm{A})-\mathrm{A} \cdot(\nabla \times \mathrm{B}))
$$

Using the divergence theorem i.e. vector form of Greens theorem over the volume $\Omega$ bounded by Periodic boundary S. Thus, the system of equations obtained are

$$
\begin{gathered}
\iiint_{\Omega} \vec{\Psi} \cdot(\nabla \times \vec{\Phi}) d V-\iiint_{S} \vec{\Psi} \cdot(v \times \vec{\Phi}) d S=-j \omega \iiint_{\Omega} \vec{\Psi} \cdot \vec{\Psi} d V \\
\iiint_{\Omega}(\nabla \times \vec{\Phi}) \cdot \vec{\Psi} d V-\mu \sigma \iiint_{\Omega} \vec{\Phi} \cdot \vec{\Phi} d V=j \omega \mu \varepsilon \iiint_{\Omega} \vec{\Phi} \cdot \vec{\Phi} d V(6)
\end{gathered}
$$

Then, the discretized linear system for the $i^{\text {th }}$ subdomain is

$$
\begin{aligned}
& S_{e b}^{i} E^{i}-B_{e b}^{i} E^{i}=j \omega M_{b b}^{i} B^{i} \\
& S_{b e}^{i} E^{i}+C_{e e}^{i} E^{i}=j \omega M_{e e}^{i} E^{i}
\end{aligned}
$$

If the elemental form of the matrices with subscripts $p$ and $q$ for the local vector indexes for basis functions are considered. The local matrices are defined as

$$
\begin{gathered}
M_{e e}^{i}=\left\langle\vec{\Phi}_{p}, \varepsilon \vec{\Phi}_{q}\right\rangle_{V_{e}} ; M_{b b}^{i}=\left\langle\vec{\Psi}_{p}, \vec{\Psi}_{q}\right\rangle_{V_{e}} \\
C_{e e}^{i}=\left\langle\vec{\Phi}_{p}, \sigma \vec{\Phi}_{q}\right\rangle_{V_{e}} ; S_{b e}^{i}=S_{e b}^{i}=\left\langle\vec{\Phi}_{p}, \nabla \times \vec{\Psi}_{q}\right\rangle_{V_{e}} \\
B_{e b}^{i}=\left\langle v \times \vec{\Phi}_{q}, \vec{\Psi}_{p}\right\rangle_{V_{e}}
\end{gathered}
$$

Here, the \langle\rangle denotes the inner product of two functions is defined as $\langle\vec{f}, \vec{g}\rangle=\int \vec{f}^{T} \cdot \vec{g} d V$, over the volume $\mathrm{V}_{\mathrm{e}}$.

\section{Floquet Decomposition}

Here for initial analysisas illustrated in Fig. 1 type of the periodic structure with periodic boundary conditions are considered for time harmonic electromagnetic fields. The structure is assumed to be periodic in the xy-plane, i.e., in $\mathrm{Z}$ direction and the $m^{\text {th }}$ cell of the array is obtained by shifting the $(0,0)$ cell through the relation.

$$
\Theta\left(x, y, z+m T_{z}\right)=\Theta(x, y, z) \cdot e^{-j m \theta_{z}}
$$

Here $\theta$ denotes the constant phase shift between two adjacent unit cells. For periodic excitation of the array with a linear phase factor, the fields in the array obey the periodicity conditions given by Floquets theorem.

$$
\begin{gathered}
\mathrm{E}\left(x, y, z+m T_{z}\right)=\mathrm{E}(x, y, z) \cdot e^{-j m \theta_{z}} \\
\mathrm{~B}\left(x, y, z+m T_{z}\right)=\mathrm{B}(x, y, z) \cdot e^{-j m\left(\theta_{z}+\pi\right)}
\end{gathered}
$$

The value of fields over the periodic boundary $\partial \Omega$ is not known. Thus an appropriate treatment for the boundary integral in (6) has to be formulated specifically, so that the value of fields on the periodic boundary is not needed in the system of equations over a unit cell. This is possible by making use of the periodic boundary conditions (10), thus reducing the system to be of independent unknowns. The application of the periodic boundary conditions through the matrix transformation $\mathrm{P}_{\mathrm{E}}$ and $\mathrm{P}_{\mathrm{B}}$ for electric and magnetic field vectors respectively as.

$$
E^{i}=P_{E} E_{\text {min }}^{i}, B^{i}=P_{B} B_{\text {min }}^{i}
$$

Here $\mathrm{E}_{\min }^{\mathrm{i}}, \mathrm{B}_{\min }^{\mathrm{i}}$ are minimised matrices and

$$
P_{E}=\left[\begin{array}{cc}
I & 0 \\
0 & I \\
0 & I e^{-j m \theta_{z}}
\end{array}\right], P_{B}=\left[\begin{array}{cc}
I & 0 \\
0 & I \\
0 & I e^{-j m\left(\theta_{z}+\pi\right)}
\end{array}\right]
$$

$I$ stands for an appropriately sized identity matrix.

\section{NUMERICAL RESULTS}

The formulation described above has been implemented and tested for several bounded and periodic geometries. The code is geometry-independent, thus meshing platform is implemented as a wrapper on top of open source mesh generator Gmsh [13]. In this section, some results are presented to demonstrate the validity and capability of the proposed method for a rectangular periodic cavity and structure illustrated in Fig. 1 and Fig. 5.

\section{A. Rectangular periodic cavity:}

The numerical example, consider is a rectangular air- filled periodic cavity with dimensions $0.1 \mathrm{~m} \times 0.05 \mathrm{~m} \times 0.2 \mathrm{~m}$ as shown in Fig. 1, with the sides in $\mathrm{Z}$ direction as periodic and all other sides as PEC boundary for the calculation domain. The existing EE method based on second order equation formulation was implemented for comparison. The convergence plots, as shown in Fig. 2, for the frequency calculated from the proposed EB method and the existing EE methods with respect to analytical result clearly suggests, that the convergence towards the actual solution is faster for EB method than the EE methods.

The present formulation results are compared with the analytical results and with reference results obtained from the commercial software [14]. The dispersion curves ( $\omega$ vs $\beta$ ) are shown in Fig. 3. Good agreement between the results are observed. It is evident from Fig. 3, that the present formulation has advantages of higher accuracy and suitability to obtain broad frequency band highly accurate results, including frequencies for the higher order Floquet mode or non-evanescent mode in propagation.

The Fig. 4 shows relative error in the calculated frequency of commercial code and proposed formulation with respect to analytical for different mesh sizes. It is evident that the dispersion curve calculated by present formulation solves with less number of mesh than the software. Table I shows, the time taken by commercial code and the proposed formulation for calculating the results are compared for the rectangular periodic cavity. Even though, the table indicates a faster solution by the commercial code for same number of meshes, the same accuracy can be achieved in the proposed formulation using less number of elements and hence the speed-up. 


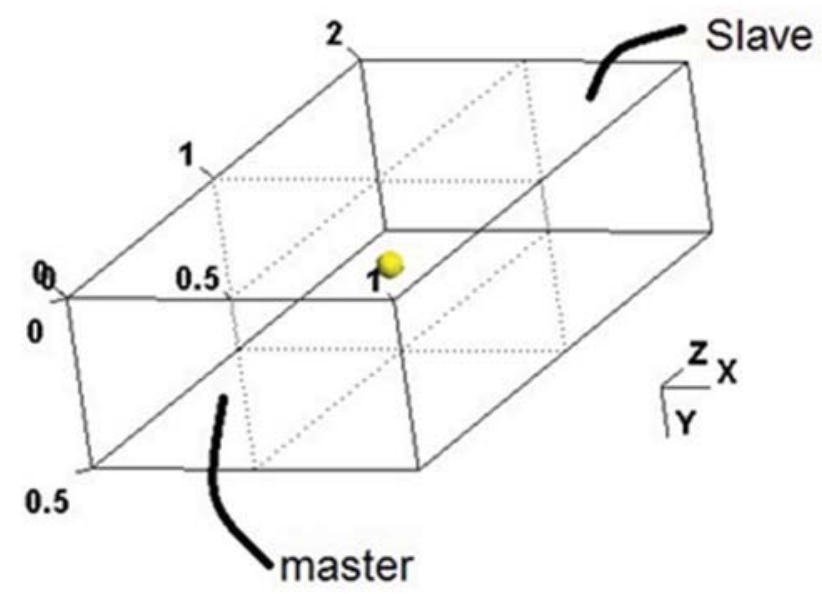

Figure 1: Rectangular air-filled periodic cavity with dimensions in $\mathrm{dm}$ $(10 \mathrm{xcm})$

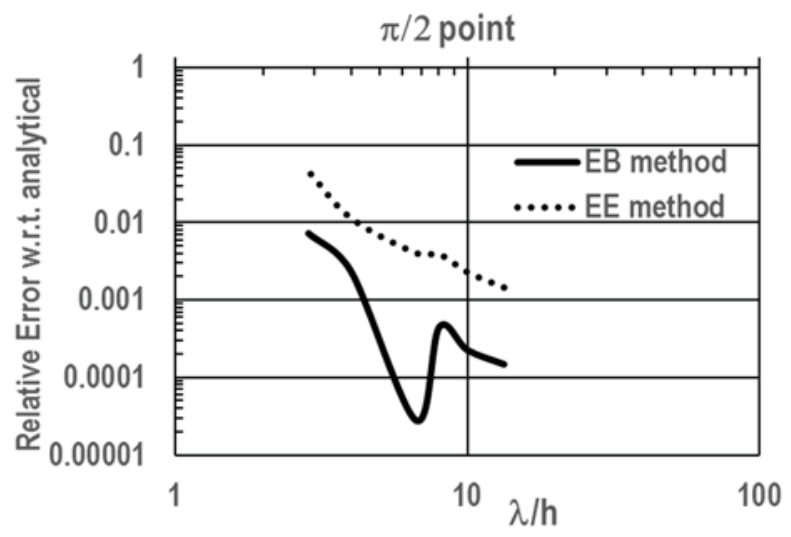

(a)

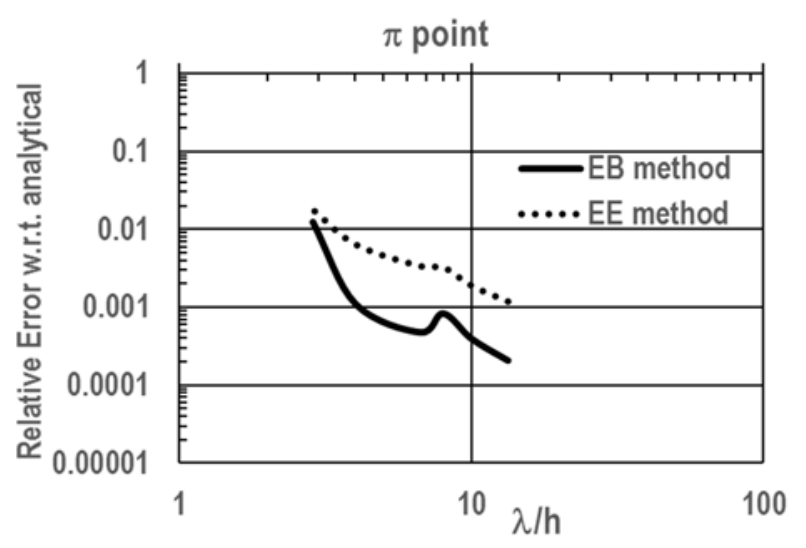

(b)

Figure 3: Comparison of relative error in dispersion calculated through proposed EB method and EE methods with respect to analytical result (a) at phase $=\pi / 2$ and (b) at phase $=\pi$

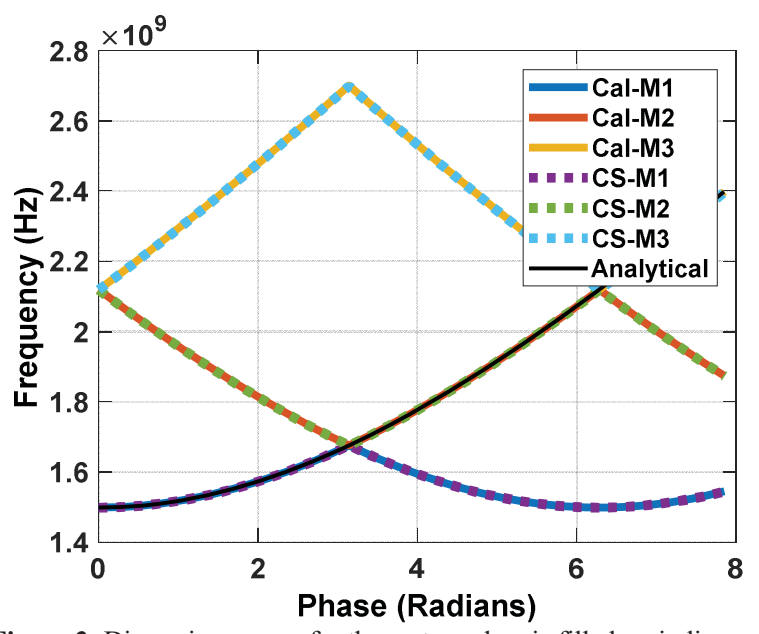

Figure 3: Dispersion curves for the rectangular air-filled periodic cavity in Fig 1; Cal-M1,Cal-M2 and Cal-M3 are calculated Floquet modes for Mode-1, Mode-2 and Mode-3 respectively, CS-M1,CS-M2 and CS-M3 are Floquet modes obtained for Mode-1, Mode- 2 and Mode- 3 respectively from commercial software and Analytical is the analytical result

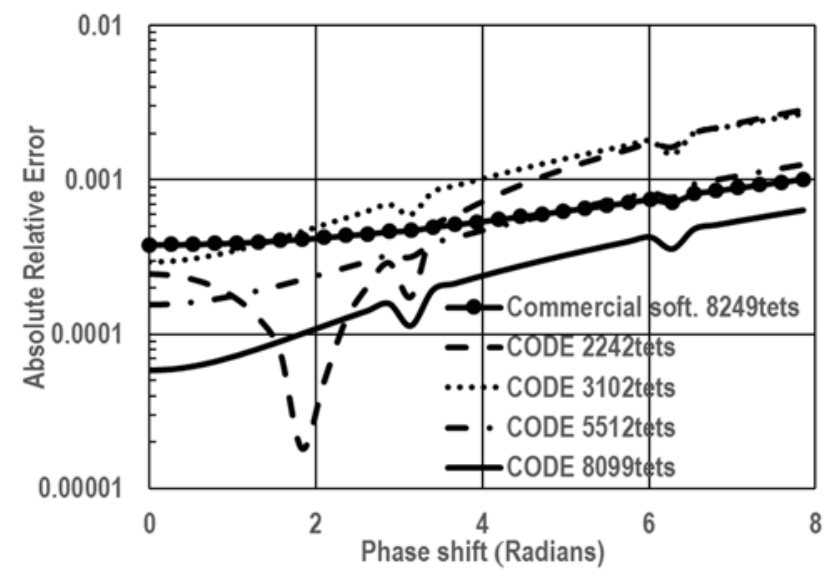

Figure 4: Comparison of relative errors in frequency calculated from commercial software and present CODE for different number of tetrahedrons(tets)

TABLE I. TIME-MEMORY SAVINGS RESULTS FOR MICROSTRIP LINE: ADAPTIVE REFINEMENT VS. UNIFORM REFINEMENT

\begin{tabular}{|c|c|c|}
\hline Mesh size & Commercial code & Present formulation \\
\hline 2242 & ---- & $31.62 \mathrm{~S}$ \\
\hline 3012 & ---- & $53.78 \mathrm{~S}$ \\
\hline 5512 & ---- & $116.5 \mathrm{~S}$ \\
\hline 8200 & $178 \mathrm{~S}$ & $254.3 \mathrm{~S}$ \\
\hline
\end{tabular}

B. Circular periodic cavity:

In the next numerical example, a circular air-filled metallic cylindrical cavity is considered with dimensions as follows: radius $=0.05 \mathrm{~m}$ and length $=0.2 \mathrm{~m}$ as shown in Fig. 5 , with the circular sides in $\mathrm{Z}$ direction as periodic and all other sides as PEC boundary for the calculation domain. The dispersion curves $(\omega$ vs $\beta)$ are shown in Fig. 6. The results from the proposed formulation with 3656 mesh elements, are compared with the analytical results and those obtained from the commercial software. The agreement between the results is good. But as shown in Fig. 7 relative error in the calculated frequency from 
commercial software with respect to analytical dispersion curve is larger to that obtained from the present formulation.

\section{CONCLUSION}

In this paper, a mixed-order FEM method is proposed for solving infinite periodic boundary value problem with complex geometry and anisotropic lossless media. The advantages of the proposed method include higher accuracy for the same number of mesh elements and suitability to obtain accurate results over a broad frequency band including frequencies for the higher order Floquet mode or nonevanescent modes.

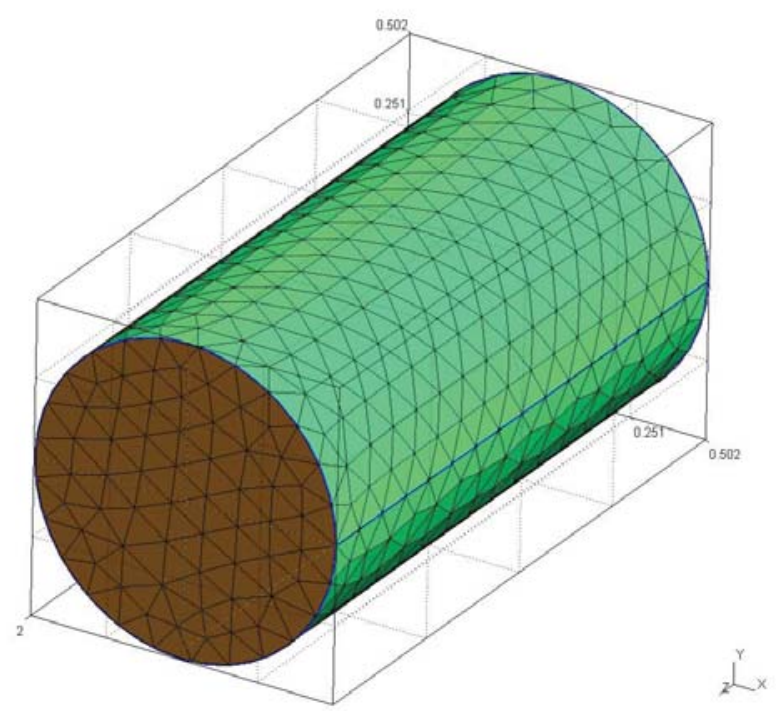

Figure 5: Cylindrical air-filled periodic cavity with dimensions in $\mathrm{dm}\left(10_{-}\right.$ $\mathrm{cm})$

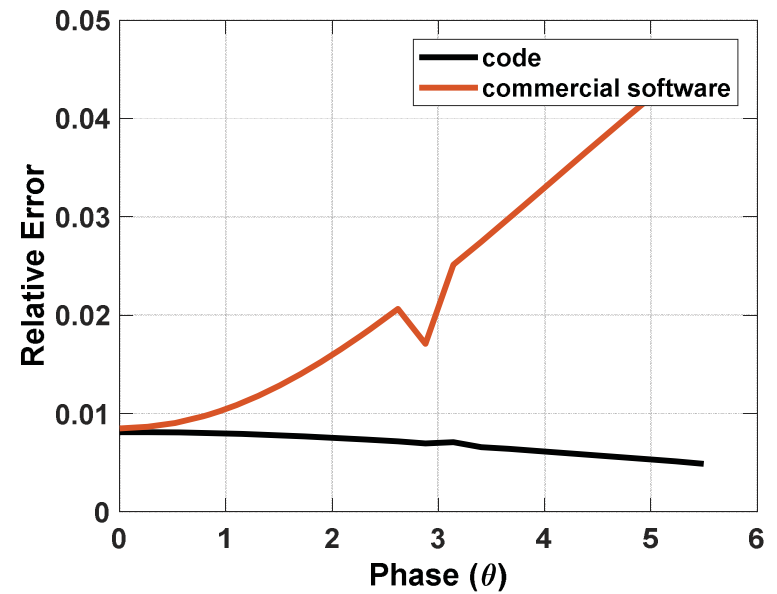

Figure. 6. Dispersion curves for the circular air-filled periodic cavity in Fig 1; Cal-M1 and Cal-M2 are calculated Floquet modes for Mode-1 and Mode-2 respectively, CS-M1 and CS-M2 are Floquet modes obtained for Mode-1 and Mode-2 respectively from Commercial csoftware and Analytical is the analytical result

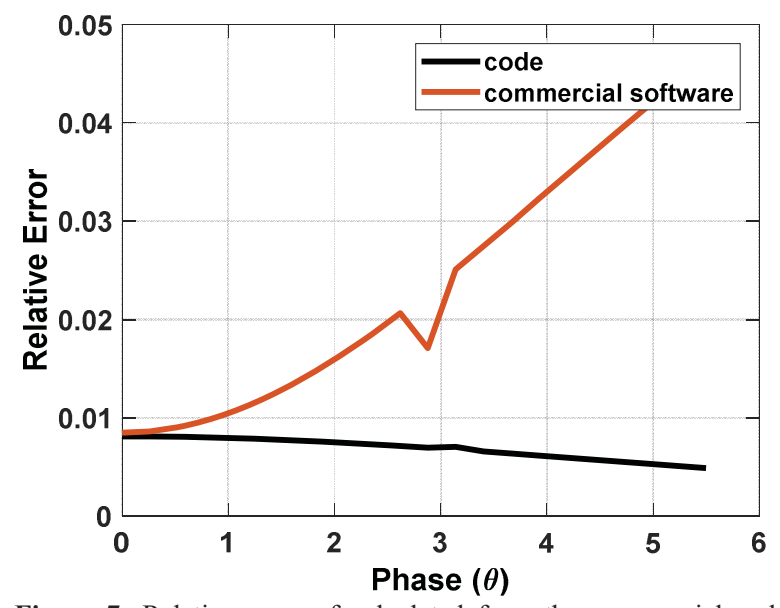

Figure 7: Relative error of calculated from the commercial code and present formulation (code) W.r.t Analytical values for Cylindrical air-filled periodic cavity

\section{REFERENCES}

[1] J. M. Jin and J. L. Volakis, "Electromagnetic scattering by a perfectly conducting patch array on a dielectric slab," IEEE Trans. Antennas Propagat., vol. 38, pp. 556-563, 1990.

[2] A. K. Bhattacharyya," Analysis of multilayer infinite periodic array structures with different periodicities and axes orientation," IEEE Trans. Antennas Propagat., vol. 48, pp. 357-369, 2000.

[3] J. M. Jin, The Finite Element Method in Electromagnetics, $3^{\text {rd }}$ Edition Hoboken, NJ: Wiley, 2014.

[4] J. M. Jin and D. J. Riley, Finite Element Analysis of Antennas and Arrays, Hoboken, NJ: Wiley, 2009.

[5] D. B. Davidson, Computational Electromagnetics for RF and Microwave Engineering, 2nd ed. New York: Cambridge University Press, Nov. 2010.

[6] P. Monk, Finite Element Methods for Maxwells Equations. Oxford,U.K.: Oxford Univ. Press, 2003.

[7] M. Wong, O. Picon, and V. F. Hanna," A finite element method based on Whitney forms to solve Maxwell equations in the time domain," IEEE Trans. Magn., vol. 31, no. 2, pp. 1618-1621, Mar. 1995.

[8] A. Bossavit, "Mixed finite elements and the complex of Whitney forms," The Mathematics of Finite Elements and Applications VI (J. Whiteman, ed.), London, U.K.: Academic, 1988, pp. 137-144.

[9] R. N. Rieben, G. H. Rodrigue, and D. A. White, "A high order mixed vector finite element method for solving the time dependent Maxwell equations on unstructured grids," J. Comp. Phys., vol. 204, pp. 490 519,2005 .

[10] B. He and F. L. Teixeira," Mixed Finite-Element Time-Domain Method for Transient Maxwell Equations in Doubly Dispersive Media," IEEE Trans. on Micro. Theory and Tech., Vol. 56, no. 1, pp. 113-119, 2008.

[11] B. He and F. L. Teixeira,"A sparse and explicit FETD via approximate inverse Hodge (mass) matrix," IEEE Microw. Wireless Compon. Lett., vol. 16 , no. 6 , pp. 348350 , Jun. 2006.

[12] A. Chatterjee, J. M. Jin and J. L. Volakis, "Computation of cavity resonances using edge-based finite elements", IEEE Trans. Microwave Theory Tech., Vol. 40, No. 11,pp. 2106-2108, 1992.

[13] C. Geuzaine, Jean-François Remacle, "Gmsh: a three-dimensional finite element mesh generator with built-in pre- and post-processing facilities",International Journal for Numerical Methods in Engineering, 2009.

[14] CST studio suite, version 2016. 